\title{
Incidence of chronic thromboembolic pulmonary hypertension after acute pulmonary embolism: a contemporary view of the published literature
}

\author{
Yvonne M. Ende-Verhaar ${ }^{1}$, Suzanne C. Cannegieter², Anton Vonk Noordegraaf ${ }^{3}$, \\ Marion Delcroix ${ }^{4}$, Piotr Pruszczyk ${ }^{5}$, Albert T.A. Mairuhu ${ }^{6}$, Menno V. Huisman $^{1}$ \\ and Frederikus A. Klok ${ }^{1}$
}

\begin{abstract}
Affiliations: 'Dept of Thrombosis and Hemostasis, Leiden University Medical Center, Leiden, The Netherlands. ${ }^{2}$ Dept of Clinical Epidemiology, Leiden University Medical Center, Leiden, The Netherlands. ${ }^{3}$ Dept of Pulmonology, VU University Medical Center, Amsterdam, The Netherlands. ${ }^{4}$ Dept of Respiratory Diseases, University Hospital of Leuven, Leuven, Belgium. ${ }^{5}$ Dept of Internal Medicine and Cardiology, Medical University of Warsaw, Warsaw, Poland. 'Dept of Vascular Medicine, Haga Hospital, The Hague, The Netherlands.
\end{abstract}

Correspondence: Y.M. Ende-Verhaar, Dept of Thrombosis and Hemostasis, LUMC (C7Q-68), Albinusdreef 2 , Postbus 9600, 2300 RC Leiden, The Netherlands. E-mail: Y.M.Ende-Verhaaralumc.nl

@ERSpublications

The incidence of CTEPH is $\mathbf{0 . 5 6} \%$ in all comers and $\sim 3 \%$ in survivors of acute pulmonary embolism http://ow.ly/PVEh306iNoD

Cite this article as: Ende-Verhaar YM, Cannegieter SC, Vonk Noordegraaf A, et al. Incidence of chronic thromboembolic pulmonary hypertension after acute pulmonary embolism: a contemporary view of the published literature. Eur Respir J 2017; 49: 1601792 [https://doi.org/10.1183/13993003.01792-2016].

ABSTRACT The incidence of chronic thromboembolic pulmonary hypertension (CTEPH) after pulmonary embolism (PE) is relevant for management decisions but is currently unknown.

We performed a meta-analysis of studies including consecutive PE patients followed for CTEPH. Study cohorts were predefined as "all comers", "survivors" or "survivors without major comorbidities". CTEPH incidences were calculated using random effects models.

We selected 16 studies totalling 4047 PE patients who were mostly followed up for $>2$-years. In 1186 all comers (two studies), the pooled CTEPH incidence was 0.56\% (95\% CI 0.1-1.0). In 999 survivors (four studies) CTEPH incidence was 3.2\% (95\% CI 2.0-4.4). In 1775 survivors without major comorbidities (nine studies), CTEPH incidence was 2.8\% (95\% CI 1.5-4.1). Both recurrent venous thromboembolism and unprovoked PE were significantly associated with a higher risk of CTEPH, with odds ratios of 3.2 (95\% CI 1.7-5.9) and 4.1 (95\% CI 2.1-8.2) respectively. The pooled CTEPH incidence in 12 studies that did not use right heart catheterisation as the diagnostic standard was $6.3 \%$ (95\% CI 4.1-8.4).

The $0.56 \%$ incidence in the all-comer group probably provides the best reflection of the incidence of CTEPH after PE on the population level. The $~ 3 \%$ incidences in the survivor categories may be more relevant for daily clinical practice. Studies that assessed CTEPH diagnosis by tests other than right heart catheterisation provide overestimated CTEPH incidences.

\footnotetext{
Earn CME accreditation by answering questions about this article. You will find these at erj.ersjournals.com/journal/cme This article has supplementary material available from erj.ersjournals.com

Received: June 012016 | Accepted after revision: Nov 052016

Conflict of interest: None declared.

Copyright @ERS 2017
} 


\section{Introduction}

Chronic thromboembolic pulmonary hypertension (CTEPH) is a distinct form of pulmonary hypertension (PH). CTEPH is believed to arise from one or multiple endothelialised pulmonary thrombi that do not resolve but lead to chronic obstruction of the pulmonary artery tree, small-vessel arteriopathy, high pulmonary vascular resistance, $\mathrm{PH}$ and progressive right heart failure [1]. Patient prognosis is very poor when CTEPH is left untreated [2]. The only curative treatment option is surgical removal of these chronic thrombi with pulmonary endarterectomy (PEA) $[1,3]$. If PEA is not feasible or fails to significantly reduce the pulmonary artery pressure, the patient's prognosis is poor. The operability of a patient depends among other factors on the presence of more advanced distal pulmonary artery remodelling, a feature that is less expected if CTEPH is diagnosed early. Also, the duration between the last pulmonary embolism (PE) and PEA was found to be a risk factor for in-hospital mortality [4]. Hence, early diagnosis is crucial for optimal treatment and a favourable outcome.

Early CTEPH diagnosis, however, has proven to be a major clinical challenge. This is demonstrated by the staggering mean diagnostic delay of 14 months demonstrated in the European CTEPH registry [5]. One explanation for this delay could be that symptoms of CTEPH are largely non-specific. Patients can even remain asymptomatic or do not mention their symptoms for months despite the presence of relevant $\mathrm{PH}$ $[1,5]$. Also, validated cost-effective CTEPH screening tools remain unavailable to date. The incidence of CTEPH after symptomatic acute PE is not exactly known and is reported to range from $0.1 \%$ to $11.8 \%$ [6-9]. More precise knowledge of the incidence of CTEPH after acute PE is clearly relevant for defining the appropriate long-term management of acute PE. An incidence of $10 \%$ or higher would certainly warrant a standardised screening protocol for CTEPH, whereas an incidence of $0.1 \%$ or lower would not.

The notable wide range in reported incidences could be caused by major differences in the selection of the studied patient populations. For instance, most studies focused on smaller subgroups of PE patients selected by the presence or absence of thrombotic risk factors, previous venous thromboembolism (VTE) and/or absence of cardiopulmonary comorbidities. In addition, and importantly, in several reports the CTEPH diagnosis was not based on the diagnostic gold standard, i.e. right heart catheterisation (RHC) [3].

We aimed to gain an accurate overview in the variety of reported incidences of CTEPH after acute PE in different patient subgroups. To do so, we conducted a systematic review and meta-analysis of the literature, focusing on those studies that applied validated diagnostic criteria of CTEPH according to current guideline recommendations [3].

\section{Methods}

Data sources and literature search

We conducted a search for all relevant publications in PubMed, MEDLINE, Embase, Web of Science, Cochrane, CINAHL, Academic Search Premier and Science Direct. We performed our search in August 2015 with a search string focusing on "chronic thromboembolic pulmonary hypertension", "pulmonary embolism", "thromboembolism", "incidence" and "risk" (supplementary material). These key words were database-specifically translated. We additionally performed a manual search of references of the identified relevant original and review articles.

\section{Study selection, data extraction and quality assessment}

Search results were combined and duplicates were removed. Studies were screened for relevance by two independent reviewers (Y. E.-V. and F.K.) on the basis of title and abstract. Discrepancies were resolved by consensus or by contacting a third reviewer (S.C.). Full-text articles or conference abstracts in the English or Dutch language identified by either reviewer as potentially relevant were retrieved for further evaluation. We did not apply any time limitations. The final selection of studies for the meta-analysis was restricted to cohort studies of patients with an objectified index diagnosis of the first or recurrent acute PE episode, who were followed for the development of CTEPH for a period of 6 months or longer, and that explicitly reported the incidence of CTEPH.

The PRISMA statement [10] was used as a basis for reporting our systematic review. Data extraction was performed by two reviewers (Y. E.-V. and F.K.). For each included study, we extracted the first author's name, the year of publication, study design (prospective or retrospective), setting of the study (single- or multicentre), the number of patients in the index cohort, the number of patients who were followed for the occurrence of CTEPH, the number of patients with a recurrent venous thromboembolic event, the number of patients with unprovoked PE, the method of selection of patients for assessment of CTEPH (all patients or only those with specific signs and symptoms), the primary test for assessment of CTEPH, the applied gold standard for CTEPH diagnosis, the total duration of follow-up and the incidence of CTEPH as reported by the authors.

For included studies, the risk of bias was evaluated in accordance with the Cochrane Collaboration's tool for assessing risk of bias and the PRISMA statement [10, 11]. We focused on the following 
criteria: 1) pre-specified study protocol, 2) clear description of inclusion and exclusion criteria, 3) inclusion of consecutive patients, 4) objectified diagnosis of PE and CTEPH based on the results of a RHC according to current guidelines [3, 12], 5) adequate anticoagulant treatment according to international standards, 6) loss to follow-up and 7) assessment of the primary endpoint in all patients. Only studies with a low risk of bias were included in the meta-analysis.

\section{Study outcomes and definitions}

Our primary aim was to determine the incidence of CTEPH after acute PE in three predefined cohort subtypes: 1) "all comers" (i.e. all consecutive patients with symptomatic PE, no exclusion criteria), 2) "survivors" (i.e. all consecutive patients with symptomatic PE who were alive after an initial treatment period of 6 months) and 3) "survivors without major comorbidity" (i.e. all consecutive patients with symptomatic PE who were alive after an initial treatment period of 6 months and did not have predefined significant cardiopulmonary, oncologic or rheumatologic comorbidities).

Our secondary aim was to determine the association of unprovoked PE and recurrent VTE with the incidence of CTEPH. Unprovoked PE was defined as VTE occurring without any of the following risk factors: major surgery or immobilisation for at least 3 days within 4 weeks preceding the PE diagnosis, active malignancy (a diagnosis of cancer within 6 months prior to enrolment, any treatment for cancer within the previous 6 months, or recurrent or metastatic cancer), a long flight (more than 6 hours) in the past 3 weeks, being pregnant or in the peripartum period, and use of oral contraceptives or hormone replacement therapy. Recurrent VTE was defined when a documented prior episode of objectified deep vein thrombosis or PE was available [12]. We additionally aimed to evaluate the method of CTEPH screening (application of a CTEPH-specific diagnostic test in all patients or only in those who displayed or reported signs and symptoms suggestive of CTEPH) on the incidence of CTEPH. To compare the reported incidences in studies that diagnosed CTEPH based on the results of a RHC, we aimed to establish the reported incidence of CTEPH after acute PE in studies in which the diagnosis of CTEPH was based on other diagnostic criteria.

\section{Statistical analysis}

The incidence was calculated by dividing the number of confirmed cases of CTEPH during follow-up by the number of patients in the cohort initially selected for screening. For the calculation of the pooled incidences of CTEPH in the three cohort subtypes, we applied a random effects model according to DerSimonian and Laird [13]. To assess the association for unprovoked PE and recurrent VTE with CTEPH, we calculated the pooled odds ratios and $95 \%$ confidence intervals for both settings, applying the same random effects model to all studies that reported the study outcomes for these subgroups separately, irrespective of the cohort subtype. We assessed heterogeneity across the various cohort studies by calculating the $\mathrm{I}^{2}$ statistic. Heterogeneity was defined as low in when $\mathrm{I}^{2}<25 \%$, as intermediate when $\mathrm{I}^{2}=25-75 \%$ and as high when $\mathrm{I}^{2}>75 \%$ [14]. The presence of publication bias was evaluated using funnel plot analysis. All analyses were performed in Stata 14.0 (Stata Corp., College Station, TX, USA).

\section{Results}

\section{Study selection}

The initial search identified 477 records in PubMed, 381 records in Medline, 555 records in EMBASE, 302 records in Web of Science, 19 records in the Cochrane Library, 36 records in CINAHL, 85 records in Academic Search Premier and 108 records in Science Direct, resulting in a total of 1062 unique references, including 170 meeting abstracts. After the first screening of the titles and abstracts, 991 records were excluded, leaving 71 for more detailed evaluation. An additional 31 studies were excluded after a full review: 18 concerned a cohort that (partly) overlapped with other cohorts identified in our search strategy, seven studies did not provide the study endpoint, three were review articles, two studies included fewer than 20 patients, and in one study the CTEPH diagnosis was based on International Classification of Diseases insurance codes. We identified one additional relevant study by reviewing the references of the included studies. Therefore, 41 studies were fully assessed for study quality (figure 1) [7, 9, 15-53]. Of those, 13 had an intermediate to high risk of bias and were thus not included in the meta-analysis. The evaluation of the quality of bias is shown in table 1 .

\section{Included studies}

All studies were cohort studies including consecutive patients with an episode of acute PE. Sixteen studies confirmed the presence of CTEPH with RHC $[7,17,18,22,27,28,30,31,33-35,38,40,44,46,50]$ and 12 applied other tests as diagnostic standard (table 1) [9, 15, 19, 21, 23, 26, 37, 41, 43, 45, 48, 49].

The 16 articles using RHC were published between 2004 [7] and 2015 [35], and included 4047 patients selected for screening (range $87-866$ per study) $[18,34]$. The follow-up duration varied between $\geqslant 3$ months 


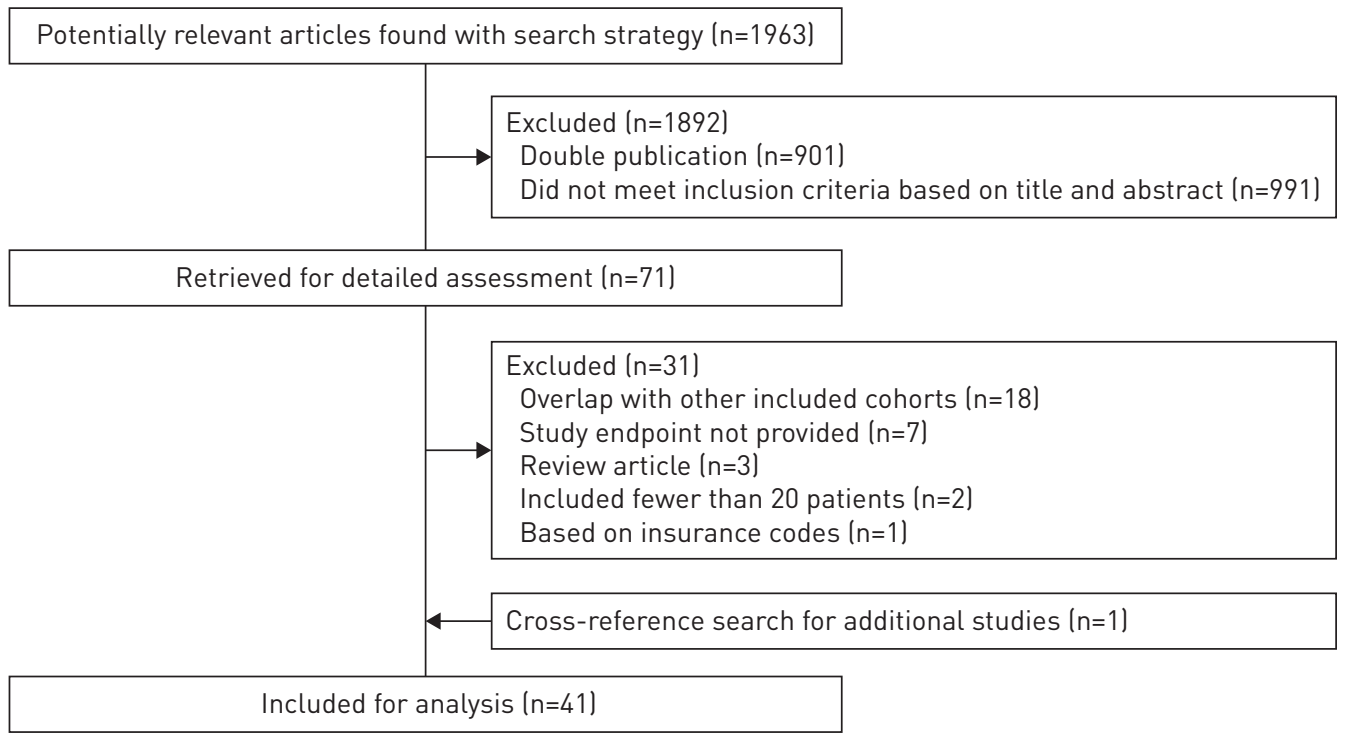

FIGURE 1 Flow diagram of study selection.

TABLE 1 Evaluation of presence of bias for all 41 identified relevant studies

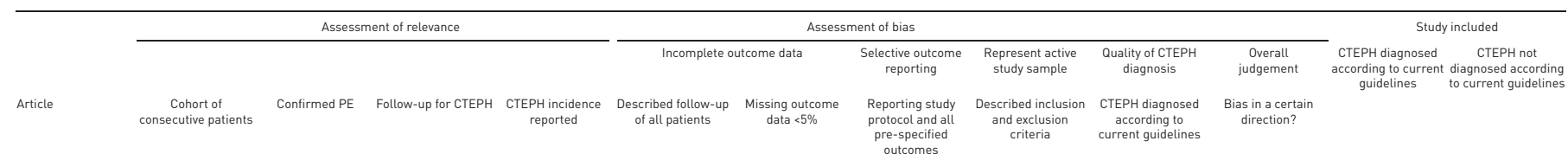

\begin{tabular}{|c|}
\hline AвuL [15] \\
\hline BaRros [16] \\
\hline BECATtinI [17] \\
\hline Berghaus [18] \\
\hline Bever-Westenoorf [19] \\
\hline CAsazza [20] \\
\hline Сно [21] \\
\hline DE FonESKa [22] $]^{\#}$ \\
\hline Dental [23] \\
\hline Dutr [24] \\
\hline FAGERBRINK [25]" \\
\hline$G_{A R Y}[26]$ \\
\hline Giulani [27] \\
\hline GoLp [9] \\
\hline GUERIN [28] \\
\hline HALL [29] \\
\hline HeLo [30]" \\
\hline HogELE [31] $]^{*}$ \\
\hline JE [32]" \\
\hline KaYAaAL [33] \\
\hline Кцок [34] \\
\hline Kเok [35] \\
\hline KoLATKIRKPANTUR [36] \\
\hline Korкмaz [37] \\
\hline MARTI [38] \\
\hline MI [39 $]^{\#+}$ \\
\hline Miniati [40] \\
\hline Otero [41] \\
\hline PaLwatwnNCHA [42]" \\
\hline Pengo [7] \\
\hline Pesavento [43]" \\
\hline PoLl [44] \\
\hline RiberRo [45] \\
\hline SURIE [46] \\
\hline Tноман [47]" \\
\hline Tosun $[48]^{*}$ \\
\hline VANNI [49]" \\
\hline VAvera [50] \\
\hline WILCZYNSKA [51] $]^{*}$ \\
\hline $\mathrm{X}_{1}[52]$ \\
\hline Yang $[53]^{\#}$ \\
\hline
\end{tabular}

\begin{tabular}{|c|c|}
\hline 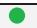 & 0 \\
\hline$\bullet$ & - \\
\hline$\bullet$ & • \\
\hline$\bullet$ & 0 \\
\hline - & • \\
\hline$\bullet$ & • \\
\hline - & 0 \\
\hline 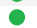 & $\$$ \\
\hline - & 0 \\
\hline • & - \\
\hline - & 8 \\
\hline 0 & 0 \\
\hline$\bullet$ & • \\
\hline$\bullet$ & - \\
\hline$\bullet$ & • \\
\hline • & - \\
\hline - & 0 \\
\hline$\bullet$ & $\S$ \\
\hline 6 & 0 \\
\hline$\bullet$ & \$ \\
\hline$\bullet$ & - \\
\hline$\bullet$ & (? \\
\hline 8 & 8 \\
\hline - & - \\
\hline 0 & 0 \\
\hline 0 & • \\
\hline$\bullet$ & - \\
\hline • & - \\
\hline 8 & 8 \\
\hline - & - \\
\hline$\bullet$ & $\bullet$ \\
\hline - & - \\
\hline 0 & $\bullet$ \\
\hline - & - \\
\hline - & \$ \\
\hline 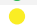 & - \\
\hline • & ${ }^{8}$ \\
\hline$\bullet$ & • \\
\hline 6 & 8 \\
\hline • & - \\
\hline$\bullet$ & $\S$ \\
\hline
\end{tabular}

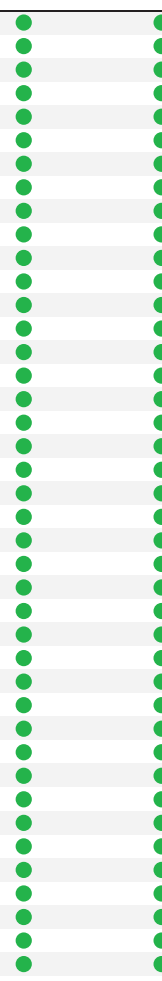

?

9

?

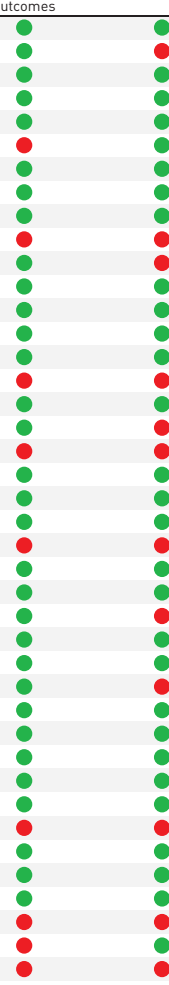

웅

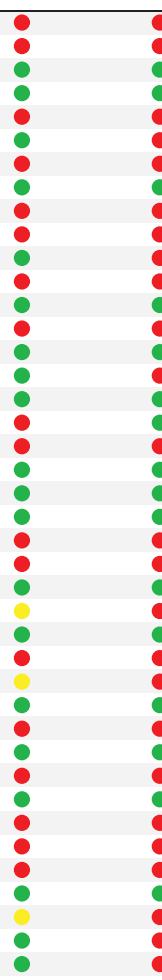

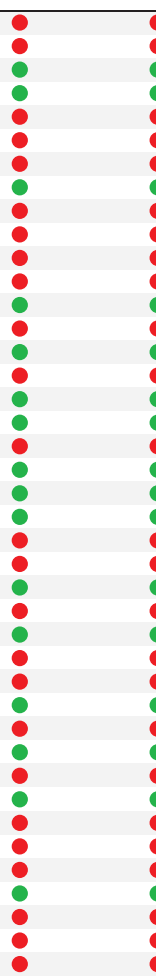

\begin{tabular}{|c|c|}
\hline - & $\boldsymbol{O}^{\prime}$ \\
\hline$\bullet$ & ○ \\
\hline - & - \\
\hline ? & 0 \\
\hline - & 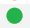 \\
\hline - & - \\
\hline - & $0^{t}$ \\
\hline - & - \\
\hline - & $\mathbf{9}^{t}$ \\
\hline - & • \\
\hline 0 & 0 \\
\hline - & - \\
\hline - & $\bullet$ \\
\hline - & • \\
\hline - & $\bullet$ \\
\hline - & - \\
\hline - & 0 \\
\hline • & • \\
\hline - & - \\
\hline - & • \\
\hline • & 0 \\
\hline - & 0 \\
\hline - & 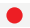 \\
\hline - & $\boldsymbol{\theta}^{t}$ \\
\hline 0 & - \\
\hline - & - \\
\hline 0 & 0 \\
\hline - & - \\
\hline - & • \\
\hline - & - \\
\hline • & $\mathbf{\theta}^{t}$ \\
\hline - & • \\
\hline ? & 0 \\
\hline - & - \\
\hline • & $\bullet$ \\
\hline - & - \\
\hline (- & ○ \\
\hline • & - \\
\hline$\bullet$ & - \\
\hline - & - \\
\hline$\bullet$ & - \\
\hline
\end{tabular}

Data are presented as the risk of bias. Green: low risk of bias; red: risk of bias; yellow: uncertain risk of bias. PE: pulmonary embolism; CTEPH: chronic thromboembolic pulmonary hypertension. \#: only the abstract was available; " study is still recruiting patients, data collection not finalised yet; ${ }^{+}$: article in Chinese; ${ }^{\S}$ : diagnostic criteria for PE not specified; ${ }^{f}$ : number of patients with abnormal echocardiography not reported. 
TABLE 2 Characteristics of the included articles for the meta-analysis of the primary endpoint

\begin{tabular}{|c|c|c|c|c|c|c|c|}
\hline & $\begin{array}{l}\text { Analysis in pro-/ } \\
\text { retrospective }\end{array}$ & Single/multicentre & $\begin{array}{l}\text { Number of patients } \\
\text { selected for screening }\end{array}$ & $\begin{array}{c}\text { Follow-up duration } \\
\text { months }\end{array}$ & Study design $\S$ & Echo criteria ${ }^{f}$ & $\begin{array}{c}\text { Number of patients } \\
\text { with CTEPH }\end{array}$ \\
\hline \multicolumn{8}{|l|}{ All comers } \\
\hline MiniATI et al. 2006 [40] & prospective & single & 320 & $0-4.8^{\#}$ & A & not specified & 4 \\
\hline KLok et al. 2010 [34] & prospective & multi & 866 & $34^{\pi}$ & $A$ & C & 4 \\
\hline \multicolumn{8}{|l|}{ Survivors } \\
\hline Hogele et al. 2014 [31] & not specified & single & 93 & 96 & $\mathrm{~B}$ & $\mathrm{D}$ & 4 \\
\hline DE FonESKa et al. 2014 [22] & retrospective & single & 616 & $\sim 3$ & $\mathrm{~B}$ & not specified & 16 \\
\hline HeLD et al. 2014 [30] & prospective & single & 130 & $3-6$ & $\mathrm{~B}$ & E & 8 \\
\hline KLok et al. 2015 [35] & prospective & single & 160 & 7 & $A$ & $\mathrm{~F}$ & 6 \\
\hline \multicolumn{8}{|l|}{ Survivors, only recurrent PE } \\
\hline Berghaus et al. 2011 [18] & retrospective & single & 87 & $22.5^{+}$ & A & G & 5 \\
\hline \multicolumn{8}{|c|}{ Survivors without major comorbidities } \\
\hline Pengo et al. 2004 [7] & prospective & single & 314 & $94^{+}$ & B & not specified & 18 \\
\hline BECATTINI et al. 2006 [17] & prospective & multi & 259 & $46^{\pi}$ & $\mathrm{B}$ & $\mathrm{H}$ & 2 \\
\hline MARTI et al. 2010 [38] & prospective & single & 294 & 24 & A & i & 10 \\
\hline PoLI et al. 2010 [44] & prospective & single & 239 & $36^{+}$ & $A$ & j & 1 \\
\hline SURIE et al. 2010 [46] & retrospective & single & 110 & $24-48$ & $\mathrm{~B}$ & $\mathrm{D}$ & 3 \\
\hline GIULIANI et al. 2014 [27] & retrospective & single & 164 & $24^{9}$ & A & 1 & 5 \\
\hline GUERIN et al. 2014 [28] & prospective & multi & 208 & $26^{+}$ & $\mathrm{B}$ & K & 7 \\
\hline KaYAaLP et al. 2014 [33] & prospective & single & 99 & $12-24$ & A & not specified & 5 \\
\hline VAVERA et al. 2014 [50] & prospective & single & 97 & 24 & A & not specified & 2 \\
\hline
\end{tabular}

CTEPH: chronic thromboembolic pulmonary hypertension; PE: pulmonary embolism. ${ }^{\#}$ : range in years; ${ }^{\text {ๆ: }}$ average; ${ }^{+}:$median; ${ }^{\S}$ : A, all consecutive patients with PE were screened for $\mathrm{CTEPH}$; $\mathrm{B}$, only patients with symptoms were screened for $\mathrm{CTEPH} ;{ }^{f} \mathrm{C}$, systolic pulmonary arterial pressure $\geqslant 35 \mathrm{mmHg}$ or mean pulmonary arterial pressure $\geqslant 25 \mathrm{mmHg}$ or four other criteria, one was needed; $\mathrm{D}$, systolic pulmonary arterial pressure $>40 \mathrm{mmHg}$; $\mathrm{E}$, right ventricular systolic pressure $\geqslant 35 \mathrm{mmHg}$; $F$, systemic pulmonary arterial pressure $>36 \mathrm{mmHg}$ or two other criteria; G, estimated pulmonary arterial systolic pressure $>50 \mathrm{mmHg} ; \mathrm{H}$, pulmonary arterial systolic pressure $>40 \mathrm{mmHg}$, pulmonary arterial pressure $>30 ;$, pulmonary arterial systolic pressure $\geqslant 40 \mathrm{mmHg}$; J, right ventricle-right atrial gradient $>35$; K: velocity of the tricuspid regurgitation $\geqslant 2.8 \mathrm{~m} \cdot \mathrm{s}^{-1}$ or proto-diastolic velocity of pulmonary regurgitation $\geqslant 2.0 \mathrm{~m} \cdot \mathrm{s}^{-1}$ and end-diastolic velocity of pulmonary regurgitation $\geqslant 1.2 \mathrm{~m} \cdot \mathrm{s}^{-1}$. 
[22] to 8 years [31], with most studies reporting a follow-up period of 2 years (table 2). The diagnostic process of selecting patients for RHC differed among the selected studies. In nine studies all included patients were screened by echocardiography $[18,27,28,33-35,38,44,50]$ and in one study all patients underwent a lung scintigraphy investigation as an initial step [40]. In five other studies, echocardiography was only performed if dyspnoea was reported by the individual patients [7, 17, 30, 31, 46]. One study applied the need for further investigation based on a not-further-defined clinical assessment prior to RHC [22]. Three studies targeted patients with a first PE (595 patients in total) $[17,44,50]$ and one study included 87 patients with recurrent VTE only [18]. The other studies focused on a combination of first and recurrent PE. The general characteristics of the studies are shown in table 2.

\section{Primary analysis: meta-analysis of incidence of CTEPH}

The overall weighted pooled incidence of CTEPH across all 16 studies was $2.3 \%$ (95\% CI 1.5-3.1; $\mathrm{I}^{2}=70.3$; figures 2 and 3). Two studies reported the CTEPH incidences in 1186 all comers who had been followed for 2-3 years $[34,40]$. The weighted pooled incidence of CTEPH in the all comers was $0.56 \%$ (95\% CI $\left.0.13-0.98 ; \mathrm{I}^{2}=98.3 \%\right)$. Four studies focused on unselected consecutive patients who were alive after an initial treatment period of at least 3 months $[22,30,31,35]$. The weighted pooled incidence of CTEPH in these 999 survivors followed for a period varying from 3 months to 8 years was $3.2 \%$ (95\% CI 2.0-4.4; $\left.\mathrm{I}^{2}=7.0 \%\right)$. One additional study in survivors included 87 patients with recurrent PE only, of whom $5.7 \%$ (95\% CI 2.5-12.8) were diagnosed with CTEPH after a 22-month follow-up period [18]. Lastly, nine studies focused on survivors without major comorbidity $[7,17,27,28,33,38,44,46,50]$. In these studies, 1775 patients were followed for 24 months or longer. Their weighted pooled incidence of CTEPH was $2.8 \%$ (95\% CI $1.5-4.1 ; \mathrm{I}^{2}=74.0 \%$ ) (figures 2 and 3). Overall, there was no difference in the incidence of CTEPH between the studies that screened all included patients versus studies that only screened patients who developed symptoms during the follow-up period. Also, a sensitivity analysis confined to studies with $\sim 2$ years of follow-up did not yield different incidences. Funnel plot analysis illustrated asymmetry, which, based on the distribution of the studies, is most likely due to between-study heterogeneity (figure 4).

\section{Secondary analysis: effect of unprovoked and recurrent PE}

In six of the 16 included articles, the incidences of CTEPH were provided for patients with unprovoked and provoked PE separately $[7,17,34,35,38,44]$. In all these studies, the incidence of CTEPH was higher after unprovoked PE versus provoked PE, for a pooled weighted OR of 4.1 (95\% CI 2.1-8.2; $\mathrm{I}^{2}=0.0 \%$ ).

Article

All comers

MINIATI et al. $2006[40]$

KLok et al. 2010 [34]

Subtotal $\left(\left.\right|^{2}=98.34 \%\right)$

Survivors

HogeLE et al. 2014 [31]

DE FONESKA et al. 2014 [22]

HeLd et al. 2014 [30]

KLok et al. 2015 [35]

Subtotal $\left(\left.\right|^{2}=2.05 \%\right)$

Survivors, only recurrent $\mathrm{PE}$

Berghaus et al. 2011 [18]

Survivors without major comorbidities

Pengo et al. 2004 [7]

BeCATtini et al. 2006 [17]

MARTI et al. 2010 [38]

Poli et al. 2010 [44]

SURIE et al. 2010 [46]

GIULIANI et al. 2014 [27]

GUERIN et al. 2014 [28]

KaYAalp et al. 2014 [33]

VAVERA et al. 2014 [50]

Subtotal $\left(\left.\right|^{2}=74.04 \%\right)$
Incidence (95\% CI) Weight \%

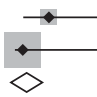

$\begin{array}{cc}1.25(0.5-3.2) & 12.1 \\ 0.46(0.2-1.2) & 87.9 \\ 0.56(0.1-1.0) & 100.0 \\ & \\ & \\ 4.3(1.7-10.5) & 8.3 \\ 2.6(1.6-4.2) & 67.5 \\ 6.2(3.2-11.7) & 8.3 \\ 3.8(1.7-7.9) & 15.9 \\ 3.2(2.0-4.4) & 100.0\end{array}$

$5.8(2.5-12.8) \quad 100.0$

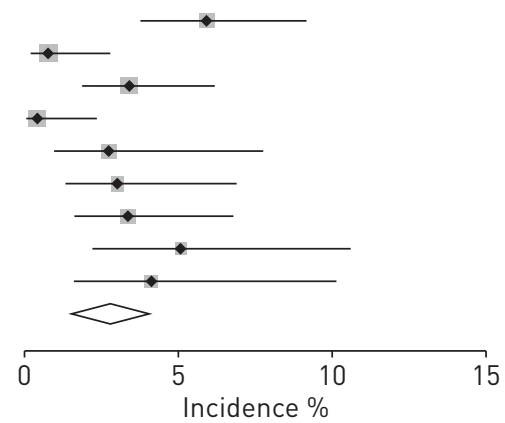

$5.9(3.8-9.1) \quad 10.5$

$0.8(0.2-2.8) \quad 16.1$

$3.4(1.9-6.1) \quad 12.5$

$0.4(0.1-2.3) \quad 16.8$

$2.7(0.9-7.7) \quad 9.2$

$3.0(1.3-6.9) \quad 10.5$

$3.4(1.6-6.8) \quad 11.2$

$5.1(2.2-11.3) \quad 6.2$

$4.1(1.6-10.1) \quad 6.9$

$2.8(1.5-4.1) \quad 100.0$

FIGURE 2 Meta-analysis of the incidences of chronic thromboembolic pulmonary hypertension after acute pulmonary embolism diagnosed with right heart catheterisation. 
FIGURE 3 Infographic of primary and secondary study outcomes. PE: pulmonary embolism.

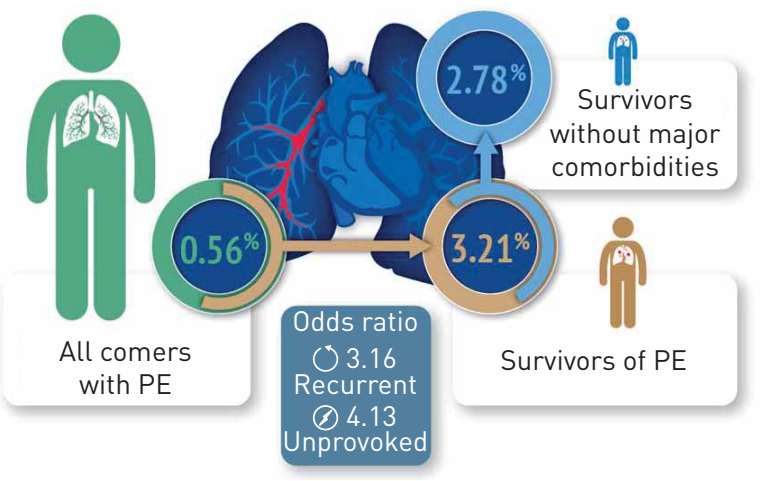

A total of seven articles allowed for the comparison of first PE versus recurrent VTE [7, 28, 34, 35, 38, 40, 46]. As with unprovoked PE, recurrent VTE was associated with a higher CTEPH incidence than after a first PE for a weighted pooled OR of 3.2 (95\% CI 1.7-5.9; $\mathrm{I}^{2}=0.0 \%$; figure 3 ).

\section{Reported incidence of CTEPH not based on RHC}

Twelve additional studies that reported the incidence of CTEPH after PE, but failed to confirm this diagnosis by RHC, were selected (table 1). The overall pooled CTEPH incidence in these studies was 6.3\% (95\% CI 4.1-8.4; $\left.\mathrm{I}^{2}=91.0 \%\right)$. In six of these 12 studies, CTEPH was diagnosed by echocardiography only, for a pooled CTEPH incidence of 9.1\% (95\% CI 4.1-14.0; $\mathrm{I}^{2}=94.4 \%$ ) (supplementary figure S1) $[9,19,26,41,45,48]$.

\section{Discussion}

This systematic review and meta-analysis summarises the existing literature on the incidence of CTEPH after acute PE. Our main findings are incidences of $0.56 \%, 3.2 \%$ and $2.8 \%$ in the three predefined subpopulations that we focused on: all comers, survivors and survivors without major comorbidities. In accordance with current knowledge [54], we identified unprovoked PE and recurrent VTE as strong risk factors for the development of CTEPH. Lastly, we showed that studies assessing the CTEPH diagnosis with tests other than RHC provide an overestimation of CTEPH incidence (pooled incidence 6.3\%), especially those using echocardiographic assessment only (pooled incidence 9.1\%).

The pooled incidence of the all comers after a follow-up period of $2-3$ years was found to be $0.56 \%$. This number represents the development of CTEPH in unselected patients after a PE diagnosis within this period and best reflects the incidence of CTEPH on a population level, mostly because no selection criteria were applied in the relevant studies. Nonetheless, several factors could have influenced this number. The incidence of $0.56 \%$ could be an underestimation caused by patients that died or were lost to follow-up without being subjected to objective tests for CTEPH. Conversely, this number could be an overestimation caused by the possibility that patients diagnosed with an acute PE had been misdiagnosed and already had CTEPH at baseline. This may be even more relevant to studies applying ventilation perfusion (VQ)-scintigraphy as the primary diagnostic test for PE, because computed tomography (CT) can show

FIGURE 4 Funnel plot analysis with the log of the number of patients with CTEPH divided by the number of patients without CTEPH. CTEPH: chronic thromboembolic pulmonary hypertension.

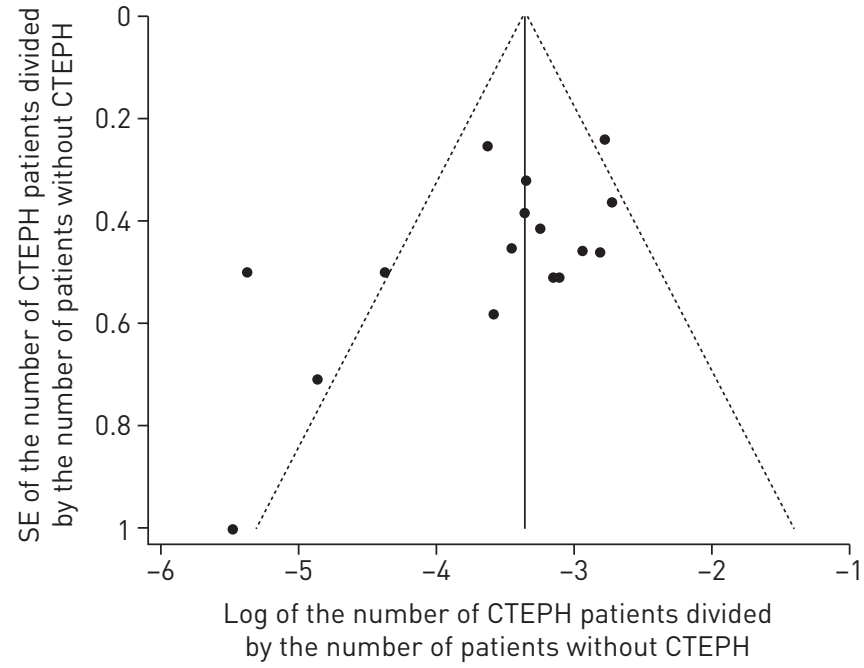


signs of $\mathrm{PH}$ that remain hidden on VQ-scintigraphy. Increasing evidence supports the hypothesis that CTEPH is often misclassified as acute $\mathrm{PE}[3,28,55,56]$. One of the included survivor studies addressed this issue with an echocardiography shortly after the PE diagnosis and a retrospective evaluation of the initial CT for signs of CTEPH at the time of the index PE. It appeared that five out of seven patients diagnosed with CTEPH already had signs of the disease at the initial presentation [28].

Epidemiological studies in CTEPH patients further support the validity of the incidence of CTEPH in the all comers we describe. Reported annual incidence rates of confirmed CTEPH are 0.9, 4.0 and 5 per million adults in the western world [57-59]. Approximately 25\% of these CTEPH patients lack a history of acute PE [5]. Considering the latter and a one-per-1000 annual rate of PE, the estimated incidence of confirmed CTEPH after acute PE ranges between 0.1 and $0.4 \%$. Of note, it is generally accepted that CTEPH is under-diagnosed in current clinical practice [60]. Taking the latter into account, the upper limit of this range is likely more accurate.

The incidence of $3.2 \%$ in the survivor cohort and $2.8 \%$ in the survivor without major comorbidities cohort may be more relevant for clinical practice because these are the patients who visit the outpatient clinic of our daily practices. With a $0.56 \%$ incidence in all comers, and considering the number of patients who died or were lost to follow-up without being subjected to objective tests for CTEPH, we expected to find an incidence of CTEPH between 1.2 and $1.8 \%$. However, we observed a five-fold higher incidence in the survivor cohorts instead. The main reason for an overestimation of the CTEPH incidence in the survivor cohorts is that patients with unprovoked PE were over-represented in the survivor cohort (48\% versus 36\% in the all-comer cohort), indicating patient selection towards a higher CTEPH risk profile. Other, less clear patient selection differences between all-comer patient cohorts and survivor patient cohorts could have further contributed to an overestimation of the CTEPH incidence in the latter, such as the exclusion of patients with high-risk PE who were not excluded from the all-comer study cohorts but were mostly excluded from the studies evaluating the survivors. Also, misclassification of acute PE at baseline as described above may be more relevant for the survivor cohorts because VQ-scintigraphy was not applied as the diagnostic test for PE in the all-comer cohorts, in contrast to the survivor cohorts. Of note, the differentiation of acute PE, CTEPH or subacute $\mathrm{PE}$ in pre-existing CTEPH is very difficult to make in clinical practice because $\mathrm{PH}$ is a common finding in acute $\mathrm{PE}$ and information on the preceding pulmonary hemodynamic status is lacking for most patients. Although no systematic search has ever been performed, perhaps extensive evaluation of the CT scans, including actively looking for webs, bands, vascular strictures, recanalised thrombi and right ventricular hypertrophy, which are all findings of CTEPH and not acute PE, as well as monitoring of the hemodynamic recovery by sequential echocardiography in the weeks after treatment initiation may help the clinician to make the distinction. This would have to be the topic of further study.

We expected to find a higher incidence of CTEPH in the survivors without major comorbidities compared to the survivors because the presence of cardiopulmonary diseases can impede optimal diagnosis of CTEPH. Nevertheless, we did not find a difference in the pooled incidence of CTEPH between survivors with or without major comorbidities (3.2\% versus $2.8 \%$ ). In a recent study of the European CTEPH registry including 679 patients diagnosed with $\mathrm{CTEPH}$, many indeed had a concomitant diagnosis of cancer $(12.7 \%)$, coronary disease and/or myocardial infarction (11.8\%) and chronic obstructive pulmonary disease (9.5\%) [5]. From this we conclude that CTEPH should be considered in all patients with CTEPH-associated symptoms despite any known other cardiopulmonary disease.

Interestingly, the reported incidences of CTEPH after acute PE in the studies that applied screening tests to all patients were not higher than in those that only screened patients who reported CTEPH-associated signs or symptoms throughout the study period. This could indicate that, although CTEPH can remain asymptomatic for months, all or almost all patients will become symptomatic at some point in the course of the disease. Notably, the studies that screened all patients and described whether the patients diagnosed with CTEPH had symptoms or not reported that all CTEPH patients had mild to severe symptoms at the moment of diagnosis, and all were diagnosed within a period of 2 years from the PE diagnosis [18, 34, 38, 44]. Based on this observation, one might argue that specific diagnostic tests for CTEPH need only be initiated when symptoms occur, as recommended by the European Society of Cardiology guideline [3]. On the other hand, the mean time to diagnosis may have been considerably shorter in the studies that screened all patients. This would support a strategy of screening patients independent of symptoms. Unfortunately, these relevant data could not be extracted from the included studies. Based on the current analysis, no firm conclusions can be made on if, in whom, when and how screening for CTEPH should optimally be performed. An algorithm that was specifically designed for this purpose, consisting of sequential application of a recently published clinical decision rule and the simple "CTEPH rule-out" criteria, is currently being evaluated in an international multicentre prospective outcome study (ClinicalTrials.gov identifier NCT02555137) [35, 61, 62]. The results of this study will allow for more accurate recommendations with regard to optimal follow-up of patients with PE on the development of CTEPH. 
Strengths of our analysis include the strict selection criteria applied, allowing for the pooling of high-quality studies with adequate diagnosis of CTEPH. We also harmonised the calculation of the CTEPH incidences using identical criteria for each study. Further, we predefined three relevant subcategories and compared studies that did or did not use RHC to diagnose CTEPH. Lastly, our finding that unprovoked PE and recurrent VTE are risk factors for CTEPH are in accordance with the literature, which underlines the validity of our work [54].

This meta-analysis has limitations as well. First, as mentioned before, CTEPH can be misclassified as acute PE $[3,28,55,56]$ although unfortunately we are not able to make this distinction in the information that was available from the studies. Of note, because CTEPH could have been present at baseline in some patients, the incidences found in our meta-analyses could actually reflect a combination of the incidence and prevalence of CTEPH. Second, the duration of follow-up varied between the included studies. Because we did not have access to patient-level data and the reporting of the follow-up time differed (means versus medians versus ranges), it was technically impossible to take individual follow-up time into account. Nonetheless, 12 of the 16 studies reported a follow-up duration $\geqslant 2$ years. As argued above, this period is likely to capture all cases of CTEPH. Third, the echocardiographic criteria for referral for RHC were slightly different across the studies, which could have induced misclassification and further patient selection. Fourth, we were not able to select the number of patients adequately treated with anticoagulants, because this was not reported in any of the studies. Inadequate anticoagulation can contribute to the development of CTEPH [63]. Fifth, despite categorising the included studies in three subgroups, we only achieved relevant inter-study homogeneity for the cohort that included survivors $\left(\mathrm{I}^{2}=7.0\right)$ [14]. The main reason this was not achieved in the all-comer cohort was the low number of two studies in this category. For the survivors without major comorbidities, this lack of homogeneity was probably caused by important differences in the definition of major comorbidities among the studies. Finally, by design, we were unable to study interesting patient groups such as those with cancer or systemic inflammatory disease [64].

In conclusion, the overall pooled incidence of CTEPH in the included studies was $2.3 \%$. The incidence of CTEPH in the all-comer cohort was low (0.56\%). This number provides the best estimation of the incidence of $\mathrm{CTEPH}$ on a population level while the $\sim 3 \%$ incidences in the survivor categories may be more relevant for daily clinical practice. Studies that assessed the CTEPH diagnosis by tests other than RHC provide overestimated CTEPH incidences.

\section{Acknowledgements}

The authors thank J.W. Schoones (the Walaeus Library of the Leiden University Medical Center, Leiden, the Netherlands) for his advice in the construction of the electronic search strategy.

Author contributions: Y.M. Ende-Verhaar was responsible for designing the study, data analysis and interpretation, and drafting of the manuscript. S.C. Cannegieter was responsible for designing the study, data analysis and interpretation, and critically revising the manuscript for important intellectual content. A. Vonk Noordegraaf, M. Delcroix, P. Pruszczyk, A.T.A. Mairuhu and M.V. Huisman were responsible for designing the study and critically revising the manuscript for important intellectual content. F.A. Klok was responsible for designing the study, data analysis and interpretation, drafting of the manuscript and supervision of the manuscript.

\section{References}

1 Lang IM, Madani M. Update on chronic thromboembolic pulmonary hypertension. Circulation 2014; 130: 508-518.

2 Riedel M, Stanek V, Widimsky J, et al. Longterm follow-up of patients with pulmonary thromboembolism. Late prognosis and evolution of hemodynamic and respiratory data. Chest 1982; 81: 151-158.

3 Galiè N, Humbert M, Vachiery JL, et al. 2015 ESC/ERS Guidelines for the diagnosis and treatment of pulmonary hypertension. The Joint Task Force for the Diagnosis and Treatment of Pulmonary Hypertension of the European Society of Cardiology (ESC) and the European Respiratory Society (ERS): Endorsed by: Association for European Paediatric and Congenital Cardiology (AEPC), International Society for Heart and Lung Transplantation (ISHLT). Eur Respir J 2015; 46: 903-975.

4 Mayer E, Jenkins D, Lindner J, et al. Surgical management and outcome of patients with chronic thromboembolic pulmonary hypertension: results from an international prospective registry. J Thorac Cardiovasc Surg 2011; 141: 702-710.

5 Pepke-Zaba J, Delcroix M, Lang I, et al. Chronic thromboembolic pulmonary hypertension (CTEPH): results from an international prospective registry. Circulation 2011; 124: 1973-1981.

6 Fedullo PF, Auger WR, Kerr KM, et al. Chronic thromboembolic pulmonary hypertension. N Engl J Med 2001; 345: 1465-1472.

7 Pengo V, Lensing AW, Prins $\mathrm{MH}$, et al. Incidence of chronic thromboembolic pulmonary hypertension after pulmonary embolism. N Engl J Med 2004; 350: 2257-2264.

8 Klok FA, Zondag W, van Kralingen KW, et al. Patient outcomes after acute pulmonary embolism. A pooled survival analysis of different adverse events. Am J Respir Crit Care Med 2010; 181: 501-506.

9 Golpe R, Perez-de-Llano LA, Castro-Anon O, et al. Right ventricle dysfunction and pulmonary hypertension in hemodynamically stable pulmonary embolism. Respir Med 2010; 104: 1370-1376.

10 PRISMA. The PRISMA statement. www.prisma-statement.org/statement.htm Date last accessed: Aug 2015. Date last updated: March 22, 2011. 
11 Higgins JPT, Altman DG, Sterne JAC, eds. Chapter 8: Assessing risk of bias in included studies. In: Higgins J Green S, eds. Cochrane Handbook for Systematic Reviews of Interventions Version 5.1.0 (updated March 2011). The Cochrane Collaboration, 2011. www.cochrane-handbook.org.

12 Huisman MV, Klok FA. Diagnostic management of acute deep vein thrombosis and pulmonary embolism. J Thromb Haemost 2013; 11: 412-422.

13 DerSimonian R, Laird N. Meta-analysis in clinical trials. Control Clin Trials 1986; 7: 177-188.

14 Higgins JP, Thompson SG, Deeks JJ, et al. Measuring inconsistency in meta-analyses. BMJ 2003; 327: 557-560.

15 Abul Y, Ozsu S, Korkmaz A, et al. Red cell distribution width: a new predictor for chronic thromboembolic pulmonary hypertension after pulmonary embolism. Chron Respir Dis 2014; 11: 73-81.

16 Barros A, Baptista R, Nogueira A, et al. Predictors of pulmonary hypertension after intermediate-to-high risk pulmonary embolism. Rev Port Cardiol 2013; 32: 857-864.

17 Becattini C, Agnelli G, Pesavento R, et al. Incidence of chronic thromboembolic pulmonary hypertension after a first episode of pulmonary embolism. Chest 2006; 130: 172-175.

18 Berghaus TM, Barac M, von Scheidt W, et al. Echocardiographic evaluation for pulmonary hypertension after recurrent pulmonary embolism. Thromb Res 2011; 128: e144-e147.

19 Beyer-Westendorf J, Weiss N, Werth S. Outpatient treatment for PE: Risk factors for mortality, recurrent VTE and pulmonary hypertension at 6 month. J Vascular Dis 2013; 42: Suppl. 84, 38.

20 Casazza F, Bongarzoni A, Forgione C, et al. Echocardiographic evolution of pulmonary artery pressure after acute pulmonary embolism. Results from IPER registry. Thromb Res 2014; 134: 1224-1228.

21 Choi J-H, Park JS, Ahn JH, et al. Incidence of chronic thromboembolic pulmonary hypertension after acute pulmonary embolism in Korea: the predictive value of echocardiography. Eur Heart J Cardiovasc Imaging 2014; 15: Suppl. 2, ii252.

22 De Foneska D, Condliffe R, Elliot CA, et al. Incidence and severity of chronic thromboembolic pulmonary hypertension following the introduction of a one-stop clinic for acute pulmonary embolism. Thorax 2014; 69: Suppl. 2, A63-A64.

23 Dentali F, Donadini M, Gianni M, et al. Incidence of chronic pulmonary hypertension in patients with previous pulmonary embolism. Thromb Res 2009; 124: 256-258.

24 Dutt TS, Mohan BV, Tousheed SZ, et al. Incidence of chronic thrombo-embolic pulmonary hypertension following acute pulmonary thrombo-embolism: an Indian perspective. Indian J Chest Dis Allied Sci 2013; 55: 205-207.

25 Fagerbrink S, Mukherjee B, Sirisena D, et al. Incidence of persistent perfusion defects following pulmonary embolism: impact on pulmonary artery systolic pressure at 6 months. Thorax 2011; 66: Suppl., A14.

26 Gary T, Starz I, Belaj K, et al. Hyperlipidemia is associated with a higher pulmonary artery systolic pressure in patients after pulmonary embolism. Thromb Res 2012; 129: 86-88.

27 Giuliani L, Piccinino C, D'Armini MA, et al. Prevalence of undiagnosed chronic thromboembolic pulmonary hypertension after pulmonary embolism. Blood Coagul Fibrinolysis 2014; 25: 649-653.

28 Guerin L, Couturaud F, Parent F, et al. Prevalence of chronic thromboembolic pulmonary hypertension after acute pulmonary embolism. Prevalence of CTEPH after pulmonary embolism. Thromb Haemost 2014; 112: 598-605.

29 Hall RJ, Sutton GC, Kerr IH. Long-term prognosis of treated acute massive pulmonary embolism. Br Heart $J$ 1977; 39: 1128-1134.

30 Held M, Hesse A, Gott F, et al. A symptom-related monitoring program following pulmonary embolism for the early detection of CTEPH: a prospective observational registry study. BMC Pulm Med 2014; $14: 141$.

31 Hogele J, Gall H, Voswinckel R, et al. Long-term screening for pulmonary hypertension after pulmonary embolism. Eur Respir J 2014; 44: Suppl. 58, P2321.

32 Jie M, Zhonghua S, Minghui Z, et al. Risk factors for chronic thromboembolic pulmonary hypertension after acute pulmonary thromboembolism. Heart 2011; 44: Suppl. 3, A227.

33 Kayaalp I, Varol Y, Cimen P, et al. The incidence of chronic thromboembolic pulmonary hypertension secondary to acute pulmonary thromboembolism. Tuberk Toraks 2014; 62: 199-206.

34 Klok FA, van Kralingen KW, van Dijk AP, et al. Prospective cardiopulmonary screening program to detect chronic thromboembolic pulmonary hypertension in patients after acute pulmonary embolism. Haematologica 2010; 95 970-975.

35 Klok FA, Tesche C, Rappold L, et al. External validation of a simple non-invasive algorithm to rule out chronic thromboembolic pulmonary hypertension after acute pulmonary embolism. Thromb Res 2015; 135: 796-801.

36 KolatKirkpantur F, Kivilcim O, Ekim N. Factors affecting the morbidity and mortality of pulmonary thromboembolism patients in long term follow-up. Chest 2004; 144: Suppl. 4, 846A.

37 Korkmaz A, Ozlu T, Ozsu S, et al. Long-term outcomes in acute pulmonary thromboembolism: the incidence of chronic thromboembolic pulmonary hypertension and associated risk factors. Clin Appl Thromb Hemost 2012; 18: 281-288.

38 Marti D, Gomez V, Escobar C, et al. Incidence of symptomatic and asymptomatic chronic thromboembolic pulmonary hypertension. Arch Bronconeumol 2010; 46: 628-633.

39 Mi J, Sun ZH, Zhong MH, et al. Predictive factors of chronic thromboembolic pulmonary hypertension in patients with acute pulmonary thromboembolism. Zhonghua Xin Xue Guan Bing Za Zhi 2012; 40: 497-501.

40 Miniati M, Monti S, Bottai M, et al. Survival and restoration of pulmonary perfusion in a long-term follow-up of patients after acute pulmonary embolism. Medicine 2006; 85: 253-262.

41 Otero R, Oribe M, Ballaz A, et al. Echocardiographic assessment of pulmonary arterial pressure in the follow-up of patients with pulmonary embolism. Thromb Res 2011; 127: 303-308.

42 Palwatwichai A, Gaewtam-Manugul P, Apiratpracha W, et al. Clinical and laboratory findings in patients with pulmonary embolism in Phramongkutklao Hospital. J Med Assoc Thai 2000; 83: 1463-1470.

43 Pesavento R, Filippi L, Palla A, et al. The incidence of chronic thromboembolic pulmonary hypertension and the predictive value of residual arterial pulmonary obstruction: Results from a large, multicentre, prospective cohort study. J Thromb Haemost 2015; 13: Suppl. 2, OR132.

44 Poli D, Grifoni E, Antonucci E, et al. Incidence of recurrent venous thromboembolism and of chronic thromboembolic pulmonary hypertension in patients after a first episode of pulmonary embolism. J Thromb Thrombolysis 2010; 30: 294-299. 
45 Ribeiro A, Lindmarker P, Johnsson $\mathrm{H}$, et al. Pulmonary embolism: one-year follow-up with echocardiography doppler and five-year survival analysis. Circulation 1999; 99: 1325-1330.

46 Surie S, Gibson NS, Gerdes VE, et al. Active search for chronic thromboembolic pulmonary hypertension does not appear indicated after acute pulmonary embolism. Thromb Res 2010; 125: e202-e205.

47 Thomas DC, Limbrey RM. Thrombolysis of acute PE patients reduces subsequent development of CTEPH. Thorax 2012; 67: Suppl. 2, A125.

48 Tosun H, Kirkil G, Deveci F, et al. The incidence of chronic thromboembolic pulmonary hypertension in asympthomatic pulmonary embolism patients. Eur Respir J 2014; 44: Suppl. 58, P2320.

49 Vanni S, Viviani G, Socci F, et al. The natural history of pulmonary hypertension in patients with acute pulmonary embolism. Eur Heart J 2010; 31: Suppl. 1, P3805.

50 Vavera Z, Vojacek J, Pudil R, et al. Chronic thromboembolic pulmonary hypertension after the first episode of pulmonary embolism? How often? Biomed PapMed Fac Univ Palacky Olomouc Czech Repub 2015; 160: 125-129.

51 Wilczynska M, Taylor K. Are we screening survivors of pulmonary embolism (PE) for chronic thromboembolic pulmonary hypertension (CTEPH)? Thorax 2011; 66: Suppl. 4, A69-A70.

52 Xi Q, Wang Y, Liu Z, et al. Red cell distribution width predicts chronic thromboembolic pulmonary hypertension in patients with acute pulmonary embolism in a long-term follow-up. Clin Chem Lab Med 2014; 52: e191-e195.

53 Yang S, Yang Y, Zhai Z, et al. Risk factors of chronic thromboembolic pulmonary hypertension after acute pulmonary embolism. Eur Respir J 2014; 44: Suppl. 58, P2324.

54 Bonderman D, Wilkens $\mathrm{H}$, Wakounig $\mathrm{S}$, et al. Risk factors for chronic thromboembolic pulmonary hypertension. Eur Respir J 2009; 33: 325-331.

55 Kim NH, Delcroix M, Jenkins DP, et al. Chronic thromboembolic pulmonary hypertension. J Am Coll Cardiol 2013; 62: D92-D99.

56 Lang IM, Pesavento R, Bonderman D, et al. Risk factors and basic mechanisms of chronic thromboembolic pulmonary hypertension: a current understanding. Eur Respir J 2013; 41: 462-468.

57 Escribano-Subias P, Blanco I, Lopez-Meseguer M, et al. Survival in pulmonary hypertension in Spain: insights from the Spanish registry. Eur Respir J 2012; 40: 596-603.

58 Hoeper MM, Humbert M, Souza R, et al. A global view of pulmonary hypertension. Lancet Respir Med 2016; 4: 306-322.

59 Delcroix M, Kerr K, Fedullo P. Chronic thromboembolic pulmonary hypertension. Epidemiology and risk factors. Ann Am Thorac Soc 2016; 13: Suppl. 3, S201-S206.

60 Hoeper MM, Madani MM, Nakanishi N, et al. Chronic thromboembolic pulmonary hypertension. Lancet Respir Med 2014; 2: 573-582.

61 Klok FA, Surie S, Kempf T, et al. A simple non-invasive diagnostic algorithm for ruling out chronic thromboembolic pulmonary hypertension in patients after acute pulmonary embolism. Thromb Res 2011; 128: 21-26.

62 Klok FA, Dzikowska-Diduch O, Kostrubiec M, et al. Derivation of a clinical prediction score for chronic thromboembolic pulmonary hypertension after acute pulmonary embolism. J Thromb Haemost 2016; 14: 121-128.

63 Konstantinides SV, Torbicki A, Agnelli G, et al. 2014 ESC guidelines on the diagnosis and management of acute pulmonary embolism. Eur Heart J 2014; 35: 3069a-3069k.

64 Lang IM, Dorfmuller P, Vonk Noordegraaf A. The pathobiology of chronic thromboembolic pulmonary hypertension. Ann Am Thorac Soc 2016; 13: Suppl. 3, S215-S221. 